Submitted to the Annals of Statistics

\title{
SUPPLEMENT TO "LIMITING LAWS OF COHERENCE OF RANDOM MATRICES WITH APPLICATIONS TO TESTING COVARIANCE STRUCTURE AND CONSTRUCTION OF COMPRESSED SENSING MATRICES"
}

\author{
By T. Tony Cai * and Tiefeng Jiang ${ }^{\dagger}$ \\ University of Pennsylvania and University of Minnesota \\ In this supplement we first give more details on Remarks 2.3 and \\ 2.4. Then we prove Propositions 4.1 and 6.2 and verify the conclusions \\ in the three examples given in Section 4, and finally we prove Lemmas \\ 6.5-6.12 which are used in the proofs of the main results.
}

Details on Remark 2.3. Consider $\Sigma=I_{p}$ with $p=2 n$ and $\tau=n$. So conditions (i) and (iii) in Theorem 4 hold, but (ii) does not. Observe

$\{(i, j) ; 1 \leq i<j \leq 2 n,|i-j| \geq n\}=n+(n-1)+\cdots+1=\frac{n(n+1)}{2} \sim \frac{p^{2}}{8}$

as $n \rightarrow \infty$. So $L_{n, \tau}$ is the maximum of roughly $p^{2} / 8$ random variables, and the dependence of any two of such random variables are less than that appeared in $L_{n}$ in Theorem 3 . The result in Theorem 3 can be rewritten as

$$
n L_{n}^{2}-2 \log \frac{p^{2}}{2}+\log \log \frac{p^{2}}{2}-\log 8 \text { converges weakly to } F
$$

as $n \rightarrow \infty$. Recalling $L_{n}$ is the maximum of roughly $p^{2} / 2$ weakly dependent random variables, replace $L_{n}$ with $L_{n, \tau}$ and $p^{2} / 2$ with $p^{2} / 8$ to have $n L_{n, \tau}^{2}-$ $2 \log \frac{p^{2}}{8}+\log \log \frac{p^{2}}{8}-\log 8$ converges weakly to $F$, where $F$ is as in Theorem 3. That is,

$$
\left(n L_{n, \tau}^{2}-4 \log p+\log \log p\right)+\log 16 \text { converges weakly to } F
$$

as $n \rightarrow \infty$ (This can be done rigorously by following the proof of Theorem 3). The difference between (76) and Theorem 4 is evident.

\footnotetext{
* The research of Tony Cai was supported in part by NSF FRG Grant DMS-0854973.

${ }^{\dagger}$ The research of Tiefeng Jiang was supported in part by NSF FRG Grant DMS0449365 .
} 
Details on Remark 2.4. Let $p=m n$ with integer $m \geq 2$. We consider the $p \times p$ matrix $\Sigma=\operatorname{diag}\left(H_{n}, \cdots, H_{n}\right)$ where there are $m H_{n}$ 's in the diagonal of $\Sigma$ and all of the entries of the $n \times n$ matrix $H_{n}$ are equal to 1 . Thus, if $\left(\zeta_{1}, \cdots, \zeta_{p}\right) \sim N_{p}(0, \Sigma)$, then $\zeta_{l n+1}=\zeta_{l n+2}=\cdots=\zeta_{(l+1) n}$ for all $0 \leq$ $l \leq m-1$ and $\zeta_{1}, \zeta_{n+1}, \cdots, \zeta_{(m-1) n+1}$ are i.i.d. $N(0,1)$-distributed random variables. Let $\left\{\zeta_{i j} ; 1 \leq i \leq n, 1 \leq j \leq m\right\}$ be i.i.d. $N(0,1)$-distributed random variables. Then

$$
(\underbrace{\zeta_{i 1}, \cdots, \zeta_{i 1}}_{n}, \underbrace{\zeta_{i 2}, \cdots, \zeta_{i 2}}_{n}, \cdots, \underbrace{\zeta_{i m}, \cdots, \zeta_{i m}}_{n})^{\prime} \in \mathbb{R}^{p}, 1 \leq i \leq n,
$$

are i.i.d. random vectors with distribution $N_{p}(0, \Sigma)$. Denote the corresponding data matrix by $\left(x_{i j}\right)_{n \times p}$. Now, take $\tau=n$ and $m=\left[e^{n^{1 / 4}}\right]$. Notice $\Gamma_{p, \delta}=p$ for any $\delta>0$. Since $p=m n$, both (i) and (ii) in Theorem 4 are satisfied, but (iii) does not. Obviously,

$$
L_{n, \tau}=\max _{1 \leq i<j \leq p,|i-j| \geq \tau}\left|\rho_{i j}\right|=\max _{1 \leq i<j \leq m}\left|\hat{\rho}_{i j}\right|,
$$

where $\hat{\rho}_{i j}$ is obtained from $\left(\zeta_{i j}\right)_{n \times m}$ as in (1) (note that the $m n$ entries of $\left(\zeta_{i j}\right)_{n \times m}$ are i.i.d. with distribution $\left.N(0,1)\right)$. By Theorem 3 on $\max _{1 \leq i<j \leq m}\left|\hat{\rho}_{i j}\right|$, we have that $n L_{n, \tau}^{2}-4 \log m+\log \log m$ converges weakly to $F$, which is the same as the $F$ in Theorem 4 . Set $\log _{2} x=\log \log x$ for $x>1$. Notice

$$
\begin{aligned}
n L_{n, \tau}^{2}-4 \log m+\log _{2} m & =n L_{n, \tau}^{2}-4 \log p+4 \log n+\log _{2} m \\
& =\left(n L_{n, \tau}^{2}-4 \log p+\log _{2} p\right)+4 \log n+o(1)
\end{aligned}
$$

since $p=m n$ and $\log _{2} p-\log _{2} m \rightarrow 0$. Further, it is easy to check that $4 \log n-16 \log _{2} p \rightarrow 0$. Therefore, the previous conclusion is equivalent to that

(77) $\left(n L_{n, \tau}^{2}-4 \log p+\log \log p\right)+16 \log \log p$ converges weakly to $F$

as $n \rightarrow \infty$. This is different from the conclusion of Theorem 4 .

Proof of Proposition 4.1. Recall the definition of $\tilde{L}_{n}$ in $(3)$, to prove the conclusion, w.l.o.g., we assume $\mu=0$ and $\sigma^{2}=1$. Evidently, by the i.i.d. assumption,

$$
\begin{aligned}
P\left(\tilde{L}_{n} \geq t\right) & \leq \frac{p^{2}}{2} P\left(\frac{\left|x_{1}^{\prime} x_{2}\right|}{\left\|x_{1}\right\| \cdot\left\|x_{2}\right\|} \geq t\right) \\
& \leq \frac{p^{2}}{2} P\left(\frac{\left|x_{1}^{\prime} x_{2}\right|}{n} \geq \frac{t}{2}\right)+\frac{p^{2}}{2} \cdot 2 P\left(\frac{\left\|x_{1}\right\|^{2}}{n} \leq \frac{1}{2}\right)
\end{aligned}
$$

imsart-aos ver. 2010/08/03 file: Coherence_Supplement_01_15_11.tex date: January 15, 2011 
where the event $\left\{\left\|x_{11}\right\|^{2} / n>1 / 2,\left\|x_{12}\right\|^{2} / n>1 / 2\right\}$ and its complement are used to get the last inequality. Since $\left\{x_{i j} ; i \geq 1, j \geq 1\right\}$ are i.i.d., the condition $E e^{t_{0}\left|x_{11}\right|^{2}}<\infty$ implies $E e^{t_{0}^{\prime}\left|x_{11} x_{12}\right|}<\infty$ for some $t_{0}^{\prime}>0$. By the Chernoff bound (see, e.g., p. 27 from Dembo and Zeitouni (1998)) and noting that $E\left(x_{11} x_{12}\right)=0$ and $E x_{11}^{2}=1$, we have

$$
P\left(\frac{\left|x_{1}^{\prime} x_{2}\right|}{n} \geq \frac{t}{2}\right) \leq 2 e^{-n I_{1}(t / 2)} \text { and } P\left(\frac{\left\|x_{1}\right\|^{2}}{n} \leq \frac{1}{2}\right) \leq 2 e^{-n I_{2}(1 / 2)}
$$

for any $n \geq 1$ and $t>0$, where the following facts about rate functions $I_{1}(x)$ and $I_{2}(y)$ are used:

(i) $I_{1}(x)=0$ if and only if $x=0 ; I_{2}(y)=0$ if and only if $y=1$;

(ii) $I_{1}(x)$ is non-decreasing on $A:=[0, \infty)$ and non-increasing on $A^{c}$. This is also true for $I_{2}(y)$ with $A=[1, \infty)$.

These and (78) conclude

$$
P\left(\tilde{L}_{n} \geq t\right) \leq p^{2} e^{-n I_{1}(t / 2)}+2 p^{2} e^{-n I_{2}(1 / 2)} \leq 3 p^{2} e^{-n g(t)}
$$

where $g(t)=\min \left\{I_{1}(t / 2), I_{2}(1 / 2)\right\}$ for any $t>0$. Obviously, $g(t)>0$ for any $t>0$ from (i) and (ii) above.

Proof of Proposition 6.2. We prove the proposition by following the outline of the proof of Proposition 6.1 step by step. It suffices to show

$$
\begin{aligned}
& \lim _{n \rightarrow \infty} P\left(\frac{W_{n}}{\sqrt{n \log p}} \geq 2+2 \epsilon\right)=0 \text { and } \\
& \lim _{n \rightarrow \infty} P\left(\frac{W_{n}}{\sqrt{n \log p}} \leq 2-\epsilon\right)=0
\end{aligned}
$$

for any $\epsilon>0$ small enough. Note that $\left|x_{11} x_{12}\right|^{\varrho}=\left|x_{11}\right|^{\varrho} \cdot\left|x_{12}\right|^{\varrho} \leq\left|x_{11}\right|^{2 \varrho}+$ $\left|x_{12}\right|^{2 \varrho}$ for any $\varrho>0$. From the given moment condition, we see that $E \exp \left(t_{0}\left|x_{11}\right|^{4 \beta /(1-\beta)}\right)<\infty$. This implies that $E \exp \left(\left|x_{11}\right|^{\frac{4 \beta}{1+\beta}}\right)<\infty$ and $E \exp \left(\left|x_{11} x_{12}\right|^{\frac{2 \beta}{1+\beta}}\right)<\infty$. By (i) of Lemma 6.4, (28) holds for $\left\{p_{n}\right\}$ such that $p_{n} \rightarrow \infty$ and $\log p_{n}=o\left(n^{\beta}\right)$. By using (27) and (29), we obtain (79).

By using condition $E \exp \left\{t_{0}\left|x_{11}\right|^{\frac{4 \beta}{1+\beta}}\right\}<\infty$ again, we know (33) also holds for $\left\{p_{n}\right\}$ such that $p_{n} \rightarrow \infty$ and $\log p_{n}=o\left(n^{\beta}\right)$. Then all statements after (30) and before (36) hold. Now, by Lemma 6.7, (37) holds for $\left\{p_{n}\right\}$ such that $p_{n} \rightarrow \infty$ and $\log p_{n}=o\left(n^{\beta}\right)$, we then have (38). This implies (30), which is the same as (80).

To verify the assertions stated in the three examples in Section 4, we need the following lemma.

imsart-aos ver. 2010/08/03 file: Coherence_Supplement_01_15_11.tex date: January 15, 2011 
LEMMA 0.1 Let $Z$ be a random variable with $E Z=0, E Z^{2}=1$ and $E e^{t_{0}|Z|}<\infty$ for some $t_{0}>0$. Choose $\alpha>0$ such that $E\left(Z^{2} e^{\alpha|Z|}\right) \leq 3 / 2$. Set $I(x)=\sup _{t \in \mathbb{R}}\left\{t x-\log E e^{t Z}\right\}$. Then $I(x) \geq x^{2} / 3$ for all $0 \leq x \leq 3 \alpha / 2$.

Proof. By the Taylor expansion, for any $x \in \mathbb{R}, e^{x}=1+x+\frac{x^{2}}{2} e^{\theta x}$ for some $\theta \in[0,1]$. It follows from $E Z=0$ that

$$
E e^{t Z}=1+\frac{t^{2}}{2} E\left(Z^{2} e^{\theta t Z}\right) \leq 1+\frac{t^{2}}{2} E\left(Z^{2} e^{t|Z|}\right) \leq 1+\frac{3}{4} t^{2}
$$

for all $0 \leq t \leq \alpha$. Use the inequality $\log (1+x) \leq x$ for all $x>-1$ to see that $\log E e^{t Z} \leq 3 t^{2} / 4$ for every $0 \leq t \leq \alpha$. Take $t_{0}=2 x / 3$ with $x>0$. Then $0 \leq t_{0} \leq \alpha$ for all $0 \leq x \leq 3 \alpha / 2$. It follows that

$$
I(x) \geq t_{0} x-\frac{3}{4} t_{0}^{2}=\frac{x^{2}}{3} .
$$

Verifications of Examples 1, 2, and 3 in Section 4. We consider the three examples one by one.

(i) If $x_{11} \sim N\left(0, n^{-1}\right)$ as in (19), then $\xi$ and $\eta$ are i.i.d. with distribution $N(0,1)$. By Lemma 3.2 from Jiang $(2005), I_{2}(x)=(x-1-\log x) / 2$ for $x>0$. So $I_{2}(1 / 2)>1 / 12$. Also, since $E e^{\theta \xi \eta}=E e^{\theta^{2} \xi^{2} / 2}=\left(1-\theta^{2}\right)^{-1 / 2}$ for $|\theta|<1$. It is straightforward to get

$$
I_{1}(x)=\frac{\sqrt{4 x^{2}+1}-1}{2}-\frac{1}{2} \log \frac{\sqrt{4 x^{2}+1}+1}{2}, \quad x>0 .
$$

Let $y=\frac{\sqrt{4 x^{2}+1}-1}{2}$. Then $y>2 x^{2} / 3$ for all $|x| \leq 4 / 5$. Thus, $I_{1}(x)=y-$ $\frac{1}{2} \log (1+y)>\frac{y}{2}>\frac{x^{2}}{3}$ for $|x| \leq 4 / 5$. Therefore, $g(t) \geq \min \left\{I_{1}\left(\frac{t}{2}\right), \frac{1}{12}\right\} \geq$ $\min \left\{\frac{t^{2}}{12}, \frac{1}{12}\right\}=\frac{t^{2}}{12}$ for $|t| \leq 1$. Since $1 /(2 k-1) \leq 1$ if $k \geq 1$. By Proposition 4.1 , we have

$$
P\left((2 k-1) \tilde{L}_{n}<1\right) \geq 1-3 p^{2} \exp \left\{-\frac{n}{12(2 k-1)^{2}}\right\}
$$

for all $n \geq 2$ and $k \geq 1$, which is (23).

(ii) Let $x_{11}$ be such that $P\left(x_{11}= \pm 1 / \sqrt{n}\right)=1 / 2$ as in (20). Then $\xi$ and $\eta$ in Proposition 4.1 are i.i.d. with $P(\xi= \pm 1)=1 / 2$. Hence, $P(\xi \eta= \pm 1)=1 / 2$ and $\xi^{2}=1$. Immediately, $I_{2}(1)=0$ and $I_{2}(x)=+\infty$ for all $x \neq 1$. If $\alpha=\log \frac{3}{2} \sim 0.405$, then $E\left(Z^{2} e^{\alpha|Z|}\right)=e^{\alpha} \leq \frac{3}{2}$ with $Z=\xi \eta$. Thus, by Lemma $0.1, I_{1}(x) \geq x^{2} / 3$ for all $0 \leq x \leq \frac{3}{5} \leq \frac{3 \alpha}{2}$. Therefore, $g(t) \geq \frac{t^{2}}{12}$ for $0 \leq t \leq \frac{6}{5}$. This gives that

$$
P\left((2 k-1) \tilde{L}_{n}<1\right) \geq 1-3 p^{2} \exp \left\{-\frac{n}{12(2 k-1)^{2}}\right\}
$$

imsart-aos ver. 2010/08/03 file: Coherence_Supplement_01_15_11.tex date: January 15, 2011 
provided $\frac{1}{2 k-1} \leq \frac{6}{5}$, that is, $k \geq \frac{11}{12}$. We then obtain (23) for all $n \geq 2$ and $k \geq 1$.

(iii) Let $x_{11}$ be such that $P\left(x_{11}= \pm \sqrt{3 / n}\right)=1 / 6$ and $P\left(x_{11}=0\right)=2 / 3$ as in (21). Then $\xi$ and $\eta$ in Proposition 4.1 are i.i.d. with $P(\xi= \pm \sqrt{3})=1 / 6$ and $P(\xi=0)=2 / 3$. It follows that $P(Z= \pm 3)=1 / 18$ and $P(Z=0)=8 / 9$ with $Z=\xi \eta$. Take $\alpha=\frac{1}{3} \log \frac{3}{2}>0.13$. Then $E\left(Z^{2} e^{\alpha|Z|}\right)=\frac{2 \times 9}{18} e^{3 \alpha}=\frac{3}{2}$. Thus, by Lemma $0.1, I_{1}(x) \geq x^{2} / 3$ for all $0 \leq x \leq \frac{3 \alpha}{2}=\frac{1}{2} \log \frac{3}{2} \sim 0.2027$. Now, $P\left(\xi^{2}=3\right)=\frac{1}{3}=1-P\left(\xi^{2}=0\right)$. Hence, $\xi^{2} / 3 \sim \operatorname{Ber}(p)$ with $p=\frac{1}{3}$. It follows that

$$
\begin{aligned}
I_{2}(x) & =\sup _{\theta \in \mathbb{R}}\left\{(3 \theta) \frac{x}{3}-\log E e^{3 \theta\left(\xi^{2} / 3\right)}\right\} \\
& =\Lambda^{*}\left(\frac{x}{3}\right)=\frac{x}{3} \log x+\left(1-\frac{x}{3}\right) \log \frac{3-x}{2}
\end{aligned}
$$

for $0 \leq x \leq 3$ by (b) of Exercise 2.2.23 from Dembo and Zeitouni (1998). Thus, $I_{2}\left(\frac{1}{2}\right)=\frac{1}{6} \log \frac{1}{2}+\frac{5}{6} \log \frac{5}{4} \sim 0.0704>\frac{1}{15}$. Now, for $0 \leq t \leq \frac{2}{5}$, we have

$$
g(t)=\min \left\{I_{1}\left(\frac{t}{2}\right), I_{2}\left(\frac{1}{2}\right)\right\} \geq \min \left\{\frac{t^{2}}{12}, \frac{1}{15}\right\}=\frac{t^{2}}{12} .
$$

Easily, $t:=\frac{1}{2 k-1} \leq \frac{2}{5}$ if and only if $k \geq \frac{7}{4}$. Thus, by Proposition 4.1,

$$
P\left((2 k-1) \tilde{L}_{n}<1\right) \geq 1-3 p^{2} \exp \left\{-\frac{n}{12(2 k-1)^{2}}\right\}
$$

for all $n \geq 2$ and $k \geq \frac{7}{4}$. We finally conclude (23) for all $n \geq 2$ and $k \geq 2$.

Proof of Lemma 6.5. (i) First, since $x_{i j}$ 's are i.i.d. bounded random variables with mean zero and variance one, by (i) of Lemma 6.4,

$$
\begin{aligned}
P\left(\sqrt{n / \log p} b_{n, 4} \geq K\right) & =P\left(\max _{1 \leq i \leq p}\left|\frac{1}{\sqrt{n \log p}} \sum_{k=1}^{n} x_{k i}\right| \geq K\right) \\
& \leq p \cdot P\left(\left|\frac{1}{\sqrt{n \log p}} \sum_{k=1}^{n} x_{k 1}\right| \geq K\right) \\
& \leq p \cdot e^{-\left(K^{2} / 3\right) \log p}=\frac{1}{p^{K^{2} / 3-1}} \rightarrow 0
\end{aligned}
$$

as $n \rightarrow \infty$ for any $K>\sqrt{3}$. This says that $\left\{\sqrt{n / \log p} b_{n, 4}\right\}$ are tight. 
Second, noticing that $|t-1| \leq\left|t^{2}-1\right|$ for any $t>0$ and $n h_{i}^{2}=\left\|x_{i}-\bar{x}_{i}\right\|^{2}=$ $x_{i}^{T} x_{i}-n\left|\bar{x}_{i}\right|^{2}$, we get that

$$
\begin{aligned}
b_{n, 1} \leq \max _{1 \leq i \leq p}\left|h_{i}^{2}-1\right| & \leq \max _{1 \leq i \leq p}\left|\frac{1}{n} \sum_{k=1}^{n}\left(x_{k i}^{2}-1\right)\right|+\max _{1 \leq i \leq p}\left|\frac{1}{n} \sum_{k=1}^{n} x_{k i}\right|^{2} \\
& =Z_{n}+b_{n, 4}^{2}
\end{aligned}
$$

where $Z_{n}=\max _{1 \leq i \leq p}\left|\frac{1}{n} \sum_{k=1}^{n}\left(x_{k i}^{2}-1\right)\right|$. Therefore,

$$
\sqrt{\frac{n}{\log p}} b_{n, 1} \leq \sqrt{\frac{n}{\log p}} Z_{n}+\sqrt{\frac{\log p}{n}} \cdot\left(\sqrt{\frac{n}{\log p}} b_{n, 4}\right)^{2} .
$$

Replacing " $x_{k i}$ " in (84) with " $x_{k i}^{2}-1$ " and using the same argument, we obtain that $\left\{\sqrt{n / \log p} Z_{n}\right\}$ are tight. Since $\log p=o(n)$ and $\left\{\sqrt{n / \log p} b_{n, 4}\right\}$ are tight, using (25) we know the second term on the right hand side of (87) goes to zero in probability as $n \rightarrow \infty$. Hence, we conclude from (87) that $\left\{\sqrt{n / \log p} b_{n, 1}\right\}$ are tight.

Finally, since $\log p=o(n)$ and $\left\{\sqrt{n / \log p} b_{n, 1}\right\}$ are tight, use (25) to have $b_{n, 1} \rightarrow 0$ in probability as $n \rightarrow \infty$. This implies that $b_{n, 3} \rightarrow 1$ in probability as $n \rightarrow \infty$.

(ii) We first claim that

$$
\begin{aligned}
& b_{n, 3} \stackrel{P}{\rightarrow} 1 \text { as } n \rightarrow \infty, \text { and }\left\{\sqrt{n / \log p} b_{n, 1}\right\} \text { and }\left\{\sqrt{n / \log p} b_{n, 4}\right\} \\
& \text { are tight if } E e^{t_{0}\left|x_{11}\right|^{\alpha}}<\infty \text { for some } 0<\alpha \leq 2 \text { and } t_{0}>0, \text { and } \\
& p_{n} \rightarrow \infty \text { and } \log p_{n}=o\left(n^{\beta_{1}}\right) \text { as } n \rightarrow \infty, \text { where } \beta_{1}=\alpha /(4-\alpha) .
\end{aligned}
$$

If the claim holds and $0<\alpha \leq 2$, recalling $\beta=\alpha /(4+\alpha)<\alpha /(4-\alpha)=\beta_{1}$, then $\log p_{n}=o\left(n^{\beta}\right)=o\left(n^{\beta_{1}}\right)$ as $n \rightarrow \infty$, the desired conclusions follow.

If claim (88) holds and $\alpha>2$, then $E e^{t_{0}\left|x_{11}\right|^{2}}<\infty$. It follows that $\left\{\sqrt{n / \log p} b_{n, 1}\right\}$ and $\left\{\sqrt{n / \log p} b_{n, 4}\right\}$ are all tight with $\log p_{n}=o(n)$. Noticing $\beta=\frac{\alpha}{4+\alpha}<1$, we see that $\left\{\sqrt{n / \log p} b_{n, 1}\right\}$ and $\left\{\sqrt{n / \log p} b_{n, 4}\right\}$ are all tight with $\log p_{n}=o\left(n^{\beta}\right)$. We also have that $b_{n, 3} \rightarrow 1$ in probability as $n \rightarrow \infty$ by the same argument as in the last paragraph of the proof of (i) above. Now we turn to prove claim (88).

By (85) and (87), to prove claim (88), it is enough to show, for some constant $K>0$,

$$
\begin{aligned}
& p \cdot P\left(\left|\frac{1}{\sqrt{n \log p}} \sum_{k=1}^{n} x_{k 1}\right| \geq K\right) \rightarrow 0 \text { and } \\
& p \cdot P\left(\left|\frac{1}{\sqrt{n \log p}} \sum_{k=1}^{n}\left(x_{k 1}^{2}-1\right)\right| \geq K\right) \rightarrow 0
\end{aligned}
$$

imsart-aos ver. 2010/08/03 file: Coherence_Supplement_01_15_11.tex date: January 15, 2011 
as $n \rightarrow \infty$. Using $a_{n}:=\sqrt{\log p_{n}}=o\left(n^{\beta / 2}\right)$ and (i) of Lemma 6.4, we have

$$
\begin{aligned}
& P\left(\left|\frac{1}{\sqrt{n \log p}} \sum_{k=1}^{n} x_{k 1}\right| \geq K\right) \leq \frac{1}{p^{K^{2} / 3}} \text { and } \\
& P\left(\left|\frac{1}{\sqrt{n \log p}} \sum_{k=1}^{n}\left(x_{k 1}^{2}-1\right)\right| \geq K\right) \leq \frac{1}{p^{K^{2} / 3}}
\end{aligned}
$$

as $n$ is sufficiently large, where the first inequality holds provided

$$
E \exp \left(t_{0}\left|x_{11}\right|^{2 \beta /(1+\beta)}\right)=E \exp \left(t_{0}\left|x_{11}\right|^{\alpha / 2}\right)<\infty ;
$$

the second holds since $E \exp \left(t_{0}\left|x_{11}^{2}-1\right|^{2 \beta /(1+\beta)}\right)=E \exp \left(t_{0}\left|x_{11}^{2}-1\right|^{\alpha / 2}\right)<\infty$ for some $t_{0}>0$, which is equivalent to $E e^{t_{0}^{\prime}\left|x_{11}\right|^{\alpha}}<\infty$ for some $t_{0}^{\prime}>0$. We then get (89) and (90) by taking $K=2$.

Proof of Lemma 6.6. Let $G_{n}=\left\{\left|\sum_{k=1}^{n} x_{k 1}^{2} / n-1\right|<\delta\right\}$. Then, by the Chernoff bound (see, e.g., p. 27 from Dembo and Zeitouni (1998)), for any $\delta \in(0,1)$, there exists a constant $C_{\delta}>0$ such that $P\left(G_{n}^{c}\right) \leq 2 e^{-n C_{\delta}}$ for all $n \geq 1$. Set $a_{n}=t_{n} \sqrt{n \log p}$. Then

$$
\Psi_{n} \leq E\left\{P^{1}\left(\left|\sum_{k=1}^{n} x_{k 1} x_{k 2}\right|>a_{n}\right)^{2} I_{G_{n}}\right\}+2 e^{-n C_{\delta}}
$$

for all $n \geq 1$. Evidently, $\left|x_{k 1} x_{k 2}\right| \leq C^{2}, E^{1}\left(x_{k 1} x_{k 2}\right)=0$ and $E^{1}\left(x_{k 1} x_{k 2}\right)^{2}=$ $x_{k 1}^{2}$, where $E^{1}$ stands for the conditional expectation given $\left\{x_{k 1}, 1 \leq k \leq n\right\}$. By the Bernstein inequality (see, e.g., p.111 from Chow and Teicher (1997)),

$$
\begin{aligned}
P^{1}\left(\left|\sum_{k=1}^{n} x_{k 1} x_{k 2}\right|>a_{n}\right)^{2} I_{G_{n}} & \leq 4 \cdot \exp \left\{-\frac{a_{n}^{2}}{\left(\sum_{k=1}^{n} x_{k 1}^{2}+C^{2} a_{n}\right)}\right\} I_{G_{n}} \\
& \leq 4 \cdot \exp \left\{-\frac{a_{n}^{2}}{\left((1+\delta) n+C^{2} a_{n}\right)}\right\} \\
& \leq \frac{1}{p^{t^{2} /(1+2 \delta)}}
\end{aligned}
$$

as $n$ is sufficiently large, since $a_{n}^{2} /\left(n(1+\delta)+C^{2} a_{n}\right) \sim t^{2}(\log p) /(1+\delta)$ as $n \rightarrow \infty$. Recalling (91), the conclusion then follows by taking $\delta$ small enough.

Proof of Lemma 6.7. Let $P^{2}$ stand for the conditional probability given $\left\{x_{k 2}, 1 \leq k \leq n\right\}$. Since $\left\{x_{i j} ; i \geq 1, j \geq 1\right\}$ are i.i.d., to prove the lemma, it 
is enough to prove

$$
\Psi_{n}:=E\left\{P^{2}\left(\left|\sum_{k=1}^{n} x_{k 1} x_{k 2}\right|>t_{n} \sqrt{n \log p}\right)^{2}\right\}=O\left(\frac{1}{p^{t^{2}-\epsilon}}\right)
$$

as $n \rightarrow \infty$. Here we use the notation " $P$ " instead of " $P$ " " simply because of the convenience of notation.

Step 1 . For any $x>0$, by the Markov inequality

$$
P\left(\max _{1 \leq k \leq n}\left|x_{k 2}\right| \geq x\right) \leq n P\left(\left|x_{12}\right| \geq x\right) \leq C n e^{-t_{0} x^{\alpha}}
$$

where $C=E e^{t_{0}\left|x_{11}\right|^{\alpha}}<\infty$. Second, we know that $E e^{t\left|x_{11}\right|^{4 \beta /(1+\beta)}}<\infty$ for any $t>0$ from the given condition. For any $\epsilon>0$, by (ii) of Lemma 6.4, there exists a constant $C=C_{\epsilon}>0$ such that

$$
P\left(\frac{\left|\sum_{k=1}^{n} x_{k 2}^{2}-n\right|}{n^{(\beta+1) / 2}} \geq \epsilon\right) \leq e^{-C_{\epsilon} n^{\beta}}
$$

for each $n \geq 1$.

Set $h_{n}=n^{(1-\beta) / 4}, \mu_{n}=E x_{i j} I\left(\left|x_{i j}\right| \leq h_{n}\right)$,

$$
\begin{aligned}
& y_{i j}=x_{i j} I\left(\left|x_{i j}\right| \leq h_{n}\right)-E x_{i j} I\left(\left|x_{i j}\right| \leq h_{n}\right) \\
& z_{i j}=x_{i j} I\left(\left|x_{i j}\right|>h_{n}\right)-E x_{i j} I\left(\left|x_{i j}\right|>h_{n}\right)
\end{aligned}
$$

for all $i \geq 1$ and $j \geq 1$. Then, $x_{i j}=y_{i j}+z_{i j}$ for all $i, j \geq 1$. Use the inequality $P(U+V \geq u+v) \leq P(U \geq u)+P(V \geq v)$ to obtain

$$
\begin{aligned}
& P^{2}\left(\left|\sum_{k=1}^{n} x_{k 1} x_{k 2}\right|>t_{n} \sqrt{n \log p}\right)^{2} \\
\leq & 2 P^{2}\left(\left|\sum_{k=1}^{n} y_{k 1} x_{k 2}\right|>\left(t_{n}-\delta\right) \sqrt{n \log p}\right)^{2} \\
& +2 P^{2}\left(\left|\sum_{k=1}^{n} z_{k 1} x_{k 2}\right|>\delta \sqrt{n \log p}\right)^{2}:=2 A_{n}+2 B_{n}
\end{aligned}
$$

for any $\delta>0$ small enough. Hence,

$$
\Psi_{n} \leq 2 E A_{n}+2 E B_{n}
$$

for all $n \geq 2$. 
Step 2: the bound of $A_{n}$. Now, if $\max _{1 \leq k \leq n}\left|x_{k 2}\right| \leq h_{n}$, then $\left|y_{k 1} x_{k 2}\right| \leq 2 h_{n}^{2}$ for all $k \geq 1$. It then follows from the Bernstein inequality (see, e.g., p. 111 from Chow and Teicher (1997)) that

$$
\begin{aligned}
A_{n} & =P^{2}\left(\left|\sum_{k=1}^{n} y_{k 1} x_{k 2}\right|>\left(t_{n}-\delta\right) \sqrt{n \log p}\right)^{2} \\
& \leq 4 \cdot \exp \left\{-\frac{\left(t_{n}-\delta\right)^{2} n \log p}{E\left(y_{11}^{2}\right) \sum_{k=1}^{n} x_{k 2}^{2}+2 h_{n}^{2}\left(t_{n}-\delta\right) \sqrt{n \log p}}\right\} \\
& \leq 4 \cdot \exp \left\{-\frac{\left(t_{n}-\delta\right)^{2} n \log p}{E\left(y_{11}^{2}\right)\left(n+\epsilon n^{(\beta+1) / 2}\right)+2 h_{n}^{2}\left(t_{n}-\delta\right) \sqrt{n \log p}}\right\}
\end{aligned}
$$

for $0<\delta<t_{n}$ and $\frac{\left|\sum_{k=1}^{n} x_{k 2}^{2}-n\right|}{n^{(\beta+1) / 2}}<\epsilon$. Notice $E\left(y_{11}^{2}\right) \rightarrow 1$ and $2 h_{n}^{2}\left(t_{n}-\right.$ $\delta) \sqrt{n \log p} / 3=o(n)$ as $n \rightarrow \infty$. Thus,

$$
\frac{\left(t_{n}-\delta\right)^{2} n \log p}{E\left(y_{11}^{2}\right)\left(n+\epsilon n^{(\beta+1) / 2}\right)+2 h_{n}^{2}\left(t_{n}-\delta\right) \sqrt{n \log p}} \sim(t-\delta)^{2} \log p
$$

as $n \rightarrow \infty$. In summary, if $\max _{1 \leq k \leq n}\left|x_{k 2}\right| \leq h_{n}$ and $\frac{\left|\sum_{k=1}^{n} x_{k 2}^{2}-n\right|}{n^{(\beta+1) / 2}} \leq \epsilon$, then for any $\delta \in(0, t / 2)$,

$$
A_{n} \leq \frac{1}{p^{t^{2}-2 t \delta}}
$$

as $n$ is sufficiently large. Therefore, for any $\epsilon>0$ small enough, take $\delta$ sufficiently small to obtain

$$
\begin{aligned}
E A_{n} & =E\left\{P^{2}\left(\left|\sum_{k=1}^{n} y_{k 1} x_{k 2}\right|>\left(t_{n}-\delta\right) \sqrt{n \log p}\right)^{2}\right\} \\
& \leq \frac{1}{p^{t^{2}-\epsilon}}+P\left(\max _{1 \leq k \leq n}\left|x_{k 2}\right| \geq h_{n}\right)+P\left(\frac{\left|\sum_{k=1}^{n} x_{k 2}^{2}-n\right|}{n^{(\beta+1) / 2}} \geq \epsilon\right) \\
& \leq \frac{1}{p^{t^{2}-\epsilon}}+C n e^{-h_{n}^{\alpha}}+e^{-C_{\epsilon} n^{\beta}}=O\left(\frac{1}{p^{t^{2}-\epsilon}}\right)
\end{aligned}
$$

as $n \rightarrow \infty$, where the second inequality follows from (94) and (95), and the last identity follows from the fact that $h_{n}^{\alpha}=n^{\beta}$ and the assumption $\log p=o\left(n^{\beta}\right)$.

Step 3: the bound of $B_{n}$. Recalling the definition of $z_{i j}$ and $\mu_{n}$ in (96), we

imsart-aos ver. 2010/08/03 file: Coherence_Supplement_01_15_11.tex date: January 15, 2011 
have

$$
\begin{aligned}
\sqrt{B_{n}}= & P^{2}\left(\left|\sum_{k=1}^{n} z_{k 1} x_{k 2}\right|>\delta \sqrt{n \log p}\right) \\
\leq & P^{2}\left(\left|\sum_{k=1}^{n} x_{k 1} x_{k 2} I\left\{\left|x_{k 1}\right|>h_{n}\right\}\right|>\delta \sqrt{n \log p} / 2\right) \\
& +I\left(\left|\sum_{k=1}^{n} x_{k 2}\right|>\frac{\delta \sqrt{n \log p}}{2\left(e^{-n}+\left|\mu_{n}\right|\right)}\right):=C_{n}+D_{n}
\end{aligned}
$$

Now, by (94),

$$
C_{n} \leq P\left(\max _{1 \leq k \leq n}\left|x_{k 1}\right|>h_{n}\right) \leq C n e^{-t_{0} h_{n}^{\alpha}}=C n e^{-t_{0} n^{\beta}} .
$$

Easily, $\left|\mu_{n}\right| \leq E\left|x_{11}\right| I\left(\left|x_{11}\right|>h_{n}\right) \leq e^{-t_{0} h_{n}^{\alpha} / 2} E\left(\left|x_{11}\right| e^{t_{0}\left|x_{11}\right|^{\alpha} / 2}\right)=C e^{-t_{0} n^{\beta} / 2}$. Also, $P\left(\left|\sum_{k=1}^{n} \eta_{k}\right| \geq x\right) \leq \sum_{k=1}^{n} P\left(\left|\eta_{k}\right| \geq x / n\right)$ for any random variables $\left\{\eta_{i}\right\}$ and $x>0$. We then have

$$
\begin{aligned}
E D_{n} & =P\left(\left|\sum_{k=1}^{n} x_{k 2}\right|>\frac{\delta \sqrt{n \log p}}{2\left(e^{-n}+\left|\mu_{n}\right|\right)}\right) \\
& \leq n P\left(\left|x_{11}\right|>\frac{\delta \sqrt{n \log p}}{2 n\left(e^{-n}+\left|\mu_{n}\right|\right)}\right) \\
& \leq n P\left(\left|x_{11}\right|>e^{t_{0} n^{\beta} / 3}\right) \leq e^{-n}
\end{aligned}
$$

as $n$ is sufficiently large, where the last inequality is from condition $E e^{t_{0}\left|x_{11}\right|^{\alpha}}<$ $\infty$. Consequently,

$$
E B_{n} \leq 2 E\left(C_{n}^{2}\right)+2 E\left(D_{n}^{2}\right)=2 E\left(C_{n}^{2}\right)+2 E\left(D_{n}\right) \leq e^{-C n^{\beta}}
$$

as $n$ is sufficiently large. This joint with (98) and (100) yields (93).

Proof of Lemma 6.8. Take $\gamma=(1-\beta) / 2 \in[1 / 3,1 / 2)$. Set

$$
\eta_{i}=\xi_{i} I\left(\left|\xi_{i}\right| \leq n^{\gamma}\right), \mu_{n}=E \eta_{1} \text { and } \sigma_{n}^{2}=\operatorname{Var}\left(\eta_{1}\right), \quad 1 \leq i \leq n
$$

Since the desired result is a conclusion about $n \rightarrow \infty$, without loss of generality, assume $\sigma_{n}>0$ for all $n \geq 1$. We first claim that there exists a constant $C>0$ such that

$$
\max \left\{\left|\mu_{n}\right|,\left|\sigma_{n}-1\right|, P\left(\left|\xi_{1}\right|>n^{\gamma}\right)\right\} \leq C e^{-n^{\beta} / C}
$$


for all $n \geq 1$. In fact, since $E \xi_{1}=0$ and $\alpha \gamma=\beta$,

$$
\begin{aligned}
\left|\mu_{n}\right|=\left|E \xi_{1} I\left(\left|\xi_{1}\right|>n^{\gamma}\right)\right| & \leq E\left|\xi_{1}\right| I\left(\left|\xi_{1}\right|>n^{\gamma}\right) \\
& \leq E\left(\left|\xi_{1}\right| e^{t_{0}\left|\xi_{1}\right|^{\alpha} / 2}\right) \cdot e^{-t_{0} n^{\beta} / 2}
\end{aligned}
$$

for all $n \geq 1$. Note that $\left|\sigma_{n}-1\right| \leq\left|\sigma_{n}^{2}-1\right|=\mu_{n}^{2}+E \xi_{1}^{2} I\left(\left|\xi_{1}\right|>n^{\gamma}\right)$, by the same argument as in (107), we know both $\left|\sigma_{n}-1\right|$ and $P\left(\left|\xi_{1}\right|>n^{\gamma}\right)$ are bounded by $C e^{-n^{\beta} / C}$ for some $C>0$. Then (106) follows.

Step 1. We prove that, for some constant $C>0$,

$$
\left|P\left(\frac{S_{n}}{\sqrt{n \log p_{n}}} \geq y_{n}\right)-P\left(\frac{\sum_{i=1}^{n} \eta_{i}}{\sqrt{n \log p_{n}}} \geq y_{n}\right)\right| \leq 2 e^{-n^{\beta} / C}
$$

for all $n \geq 1$. Observe

$$
\xi_{i} \equiv \eta_{i} \text { for } 1 \leq i \leq n \text { if } \max _{1 \leq i \leq n}\left|\xi_{i}\right| \leq n^{\gamma}
$$

Then, by (106),

$$
\begin{aligned}
P\left(\frac{S_{n}}{\sqrt{n \log p_{n}}} \geq y_{n}\right) & \leq P\left(\frac{S_{n}}{\sqrt{n \log p_{n}}} \geq y_{n}, \max _{1 \leq i \leq n}\left|\xi_{i}\right| \leq n^{\gamma}\right)+P\left(\bigcup_{i=1}^{n}\left\{\left|\xi_{i}\right|>n^{\gamma}\right\}\right) \\
(110) & \leq P\left(\frac{\sum_{i=1}^{n} \eta_{i}}{\sqrt{n \log p_{n}}} \geq y_{n}\right)+C n e^{-n^{\beta} / C}
\end{aligned}
$$

for all $n \geq 1$. Use inequality that $P(A B) \geq P(A)-P\left(B^{c}\right)$ for any events $A$ and $B$ to have

$$
\begin{aligned}
P\left(\frac{S_{n}}{\sqrt{n \log p_{n}}} \geq y_{n}\right) & \geq P\left(\frac{S_{n}}{\sqrt{n \log p_{n}}} \geq y_{n}, \max _{1 \leq i \leq n}\left|\xi_{i}\right| \leq n^{\gamma}\right) \\
& =P\left(\frac{\sum_{i=1}^{n} \eta_{i}}{\sqrt{n \log p_{n}}} \geq y_{n}, \max _{1 \leq i \leq n}\left|\xi_{i}\right| \leq n^{\gamma}\right) \\
& \geq P\left(\frac{\sum_{i=1}^{n} \eta_{i}}{\sqrt{n \log p_{n}}} \geq y_{n}\right)-C n e^{-n^{\beta} / C}
\end{aligned}
$$

where in the last step the inequality $P\left(\max _{1 \leq i \leq n}\left|\xi_{i}\right|>n^{\gamma}\right) \leq C n e^{-n^{\beta} / C}$ is used as in (110). This and (110) concludes (108).

Step 2. Now we prove

$$
P\left(\frac{\sum_{i=1}^{n} \eta_{i}}{\sqrt{n \log p_{n}}} \geq y_{n}\right) \sim \frac{e^{-x_{n}^{2} / 2}}{\sqrt{2 \pi} x_{n}}
$$


as $n \rightarrow \infty$, where

$$
x_{n}=y_{n}^{\prime} \sqrt{\log p_{n}} \text { and } y_{n}^{\prime}=\frac{1}{\sigma_{n}}\left(y_{n}-\sqrt{\frac{n}{\log p_{n}}} \mu_{n}\right) .
$$

First, by (106),

$$
\left|y_{n}^{\prime}-y_{n}\right| \leq \frac{\left|1-\sigma_{n}\right|}{\sigma_{n}} y_{n}+\frac{1}{\sigma_{n}} \cdot \sqrt{\frac{n}{\log p_{n}}}\left|\mu_{n}\right| \leq C e^{-n^{\beta} / C}
$$

for all $n \geq 1$ since both $\sigma_{n}$ and $y_{n}$ have limits and $p_{n} \rightarrow \infty$. In particular, since $\log p_{n}=o\left(n^{\beta}\right)$,

$$
x_{n}=o\left(n^{\beta / 2}\right)
$$

as $n \rightarrow \infty$. Now, set

$$
\eta_{i}^{\prime}=\frac{\eta_{i}-\mu_{n}}{\sigma_{n}}
$$

for $1 \leq i \leq n$. Easily

$$
P\left(\frac{\sum_{i=1}^{n} \eta_{i}}{\sqrt{n \log p_{n}}} \geq y_{n}\right)=P\left(\frac{\sum_{i=1}^{n} \eta_{i}^{\prime}}{\sqrt{n \log p_{n}}} \geq y_{n}^{\prime}\right)
$$

for all $n \geq 1$. Reviewing (105), for some constant $K>0$, we have $\left|\eta_{i}^{\prime}\right| \leq K n^{\gamma}$ for $1 \leq i \leq n$. Take $c_{n}=K n^{\gamma-1 / 2}$. Recalling $x_{n}$ in (112). It is easy to check that

$$
\begin{aligned}
& s_{n}:=\left(\sum_{i=1}^{n} E \eta_{i}^{\prime 2}\right)^{1 / 2}=\sqrt{n}, \varrho_{n}:=\sum_{i=1}^{n} E\left|\eta_{i}^{\prime}\right|^{3} \sim n C, \quad\left|\eta_{i}^{\prime}\right| \leq c_{n} s_{n} \text { and } \\
& 0<c_{n} \leq 1
\end{aligned}
$$

as $n$ is sufficiently large. Recall $\gamma=(1-\beta) / 2$, it is easy to see from (114) that

$$
0<x_{n}<\frac{1}{18 c_{n}}
$$

for $n$ large enough. Now, let $\gamma(x)$ be as in Lemma 6.3 , since $\beta \leq 1 / 3$, by the lemma and (114),

$$
\left|\gamma\left(\frac{x_{n}}{s_{n}}\right)\right| \leq \frac{2 x_{n}^{3} \varrho_{n}}{s_{n}^{3}}=o\left(n^{\frac{3 \beta}{2}-\frac{1}{2}}\right) \rightarrow 0 \text { and } \frac{\left(1+x_{n}\right) \varrho_{n}}{s_{n}^{3}}=O\left(n^{(\beta-1) / 2}\right) \rightarrow 0
$$


as $n \rightarrow \infty$. By (112) and (113), $x_{n} s_{n}=y_{n}^{\prime} \sqrt{n \log p_{n}}$ and $x_{n} \rightarrow \infty$ as $n \rightarrow \infty$. Use Lemma 6.3 and the fact $1-\Phi(t)=\frac{1}{\sqrt{2 \pi} t} e^{-t^{2} / 2}$ as $t \rightarrow+\infty$ to obtain

$$
P\left(\frac{\sum_{i=1}^{n} \eta_{i}^{\prime}}{\sqrt{n \log p_{n}}} \geq y_{n}^{\prime}\right)=P\left(\sum_{i=1}^{n} \eta_{i}^{\prime} \geq x_{n} s_{n}\right) \sim 1-\Phi\left(x_{n}\right) \sim \frac{e^{-x_{n}^{2} / 2}}{\sqrt{2 \pi} x_{n}}
$$

as $n \rightarrow \infty$. This and (115) conclude (111).

Step 3. Now we show

$$
\frac{e^{-x_{n}^{2} / 2}}{\sqrt{2 \pi x_{n}}} \sim \frac{p_{n}^{-y_{n}^{2} / 2}\left(\log p_{n}\right)^{-1 / 2}}{\sqrt{2 \pi} y}:=\omega_{n}
$$

as $n \rightarrow \infty$. Since $y_{n} \rightarrow y$ and $\sigma_{n} \rightarrow 1$, we know from (113) that

$$
\sqrt{2 \pi} x_{n}=\sqrt{2 \pi} y_{n}^{\prime}\left(\log p_{n}\right)^{1 / 2} \sim \sqrt{2 \pi} y\left(\log p_{n}\right)^{1 / 2}
$$

as $n \rightarrow \infty$. Further, by (112),

$$
\frac{e^{-x_{n}^{2} / 2}}{p_{n}^{-y_{n}^{2} / 2}}=\exp \left\{-\frac{x_{n}^{2}}{2}+\frac{y_{n}^{2}}{2} \log p_{n}\right\}=\exp \left\{\frac{1}{2}\left(y_{n}^{2}-y_{n}^{\prime 2}\right) \log p_{n}\right\}
$$

Since $y_{n} \rightarrow y$, by (113), both $\left\{y_{n}\right\}$ and $\left\{y_{n}^{\prime}\right\}$ are bounded. It follows from (113) again that $\left|y_{n}^{2}-y_{n}^{\prime 2}\right| \leq C\left|y_{n}-y_{n}^{\prime}\right|=O\left(e^{-n^{\beta} / C}\right)$ as $n \rightarrow \infty$. With assumption $\log p_{n}=o\left(n^{\beta}\right)$ we get $e^{-x_{n}^{2} / 2} \sim p_{n}^{-y_{n}^{2} / 2}$ as $n \rightarrow \infty$, which combining with (118) yields (117).

Finally, we compare the right hand sides of (108) and (117). Choose $C^{\prime}>$ $\max \left\{y_{n}^{2} ; n \geq 1\right\}$, since $\log p_{n}=o\left(n^{\beta}\right)$, recall $\omega_{n}$ in (117),

$$
\begin{aligned}
\frac{2 e^{-n^{\beta} / C}}{\omega_{n}} & =2 \sqrt{2 \pi} y\left(\log p_{n}\right)^{1 / 2} p_{n}^{y_{n}^{2} / 2} e^{-n^{\beta} / C} \\
& =O\left(n^{\beta / 2} \cdot \exp \left\{C^{\prime} \log p_{n}-\frac{n^{\beta}}{C}\right\}\right) \\
& =O\left(n^{\beta / 2} \cdot \exp \left\{-\frac{n^{\beta}}{2 C}\right\}\right) \rightarrow 0
\end{aligned}
$$

as $n \rightarrow \infty$ for any constant $C>0$. This fact joint with (108), (111) and (117) proves the lemma.

Proof of Lemma 6.9. For any Borel set $A \subset \mathbb{R}$, set

$$
P_{2}(A)=P\left(A \mid u_{k 1}, u_{k 3}, 1 \leq k \leq n\right),
$$

imsart-aos ver. 2010/08/03 file: Coherence_Supplement_01_15_11.tex date: January 15, 2011 
the conditional probability of $A$ with respect to $u_{k 1}, u_{k 3}, 1 \leq k \leq n$. Observe from the expression of $\Sigma_{4}$ that three sets of random variables $\left\{u_{k 1}, u_{k 3} ; 1 \leq\right.$ $k \leq n\},\left\{u_{k 2} ; 1 \leq k \leq n\right\}$ and $\left\{u_{k 4} ; 1 \leq k \leq n\right\}$ are independent. Then

$$
\begin{aligned}
& P\left(\left|\sum_{k=1}^{n} u_{k 1} u_{k 2}\right|>a_{n},\left|\sum_{k=1}^{n} u_{k 3} u_{k 4}\right|>a_{n}\right) \\
= & E\left\{P_{2}\left(\left|\sum_{k=1}^{n} u_{k 1} u_{k 2}\right|>a_{n}\right) P_{2}\left(\left|\sum_{k=1}^{n} u_{k 3} u_{k 4}\right|>a_{n}\right)\right\} \\
\leq & \left\{E P_{2}\left(\left|\sum_{k=1}^{n} u_{k 1} u_{k 2}\right|>a_{n}\right)^{2}\right\}^{1 / 2} \cdot\left\{E P_{2}\left(\left|\sum_{k=1}^{n} u_{k 3} u_{k 4}\right|>a_{n}\right)^{2}\right\}^{1 / 2}
\end{aligned}
$$

by the Cauchy-Schwartz inequality. Use the same independence again

$$
\begin{aligned}
& P_{2}\left(\left|\sum_{k=1}^{n} u_{k 1} u_{k 2}\right|>a_{n}\right)=P\left(\left|\sum_{k=1}^{n} u_{k 1} u_{k 2}\right|>a_{n} \mid u_{k 1}, 1 \leq k \leq n\right) \\
& P_{2}\left(\left|\sum_{k=1}^{n} u_{k 3} u_{k 4}\right|>a_{n}\right)=P\left(\left|\sum_{k=1}^{n} u_{k 3} u_{k 4}\right|>a_{n} \mid u_{k 3}, 1 \leq k \leq n\right) .
\end{aligned}
$$

These can be also seen from Proposition 27 in Fristedt and Gray (1997). It follows that

$$
\begin{aligned}
& \sup _{|r| \leq 1} P\left(\left|\sum_{k=1}^{n} u_{k 1} u_{k 2}\right|>a_{n},\left|\sum_{k=1}^{n} u_{k 3} u_{k 4}\right|>a_{n}\right) \\
\leq & E\left\{P\left(\left|\sum_{k=1}^{n} u_{k 1} u_{k 2}\right|>a_{n} \mid u_{11}, \cdots, u_{n 1}\right)^{2}\right\} .
\end{aligned}
$$

Since $\left\{u_{k 1} ; 1 \leq k \leq n\right\}$ and $\left\{u_{k 2} ; 1 \leq k \leq n\right\}$ are independent, and $t_{n}:=a_{n} / \sqrt{n \log p} \rightarrow t=2$, taking $\alpha=2$ in Lemma 6.7 , we obtain the desired conclusion from the lemma.

Proof of Lemma 6.10. Since $\Sigma_{4}$ is always non-negative definite, the determinant of the first $3 \times 3$ minor of $\Sigma_{4}$ is non-negative: $1-r_{1}^{2}-r_{2}^{2} \geq 0$. Let $r_{3}=\sqrt{1-r_{1}^{2}-r_{2}^{2}}$ and $\left\{u_{k 5} ; 1 \leq k \leq n\right\}$ be i.i.d. standard normals which are independent of $\left\{u_{k i} ; 1 \leq i \leq 4 ; 1 \leq k \leq n\right\}$. Then,

$$
\left(u_{11}, u_{12}, u_{13}, u_{14}\right) \stackrel{d}{=}\left(u_{11}, u_{12}, r_{1} u_{11}+r_{2} u_{12}+r_{3} u_{15}, u_{14}\right) .
$$


Define $Z_{i j}=\left|\sum_{k=1}^{n} u_{k i} u_{k j}\right|$ for $1 \leq i, j \leq 5$ and $r_{5}=r_{3}$. By the CauchySchwartz inequality,

$$
\begin{aligned}
\left|\sum_{k=1}^{n}\left(r_{1} u_{k 1}+r_{2} u_{k 2}+r_{3} u_{k 5}\right) u_{k 4}\right| & \leq \sum_{i \in\{1,2,5\}}\left|r_{i}\right| \cdot\left|\sum_{k=1}^{n} u_{k i} u_{k 4}\right| \\
& \leq\left(r_{1}^{2}+r_{2}^{2}+r_{3}^{2}\right)^{1 / 2}\left(Z_{14}^{2}+Z_{24}^{2}+Z_{54}^{2}\right)^{1 / 2} \\
& \leq \sqrt{3} \cdot \max \left\{Z_{14}, Z_{24}, Z_{54}\right\}
\end{aligned}
$$

It follows from the above two facts that

$$
\begin{aligned}
& P\left(\left|\sum_{k=1}^{n} u_{k 1} u_{k 2}\right|>a_{n},\left|\sum_{k=1}^{n} u_{k 3} u_{k 4}\right|>a_{n}\right) \\
\leq & P\left(Z_{12}>a_{n}, \max \left\{Z_{14}, Z_{24}, Z_{54}\right\}>\frac{a_{n}}{\sqrt{3}}\right) \\
\leq & \sum_{i \in\{1,2,5\}} P\left(Z_{12}>a_{n}, Z_{i 4}>\frac{a_{n}}{\sqrt{3}}\right) \\
(122)= & 2 P\left(Z_{12}>a_{n}, Z_{14}>\frac{a_{n}}{\sqrt{3}}\right)+P\left(Z_{12}>a_{n}\right) \cdot P\left(Z_{54}>\frac{a_{n}}{\sqrt{3}}\right)
\end{aligned}
$$

by symmetry and independence. For any Borel set $A \subset \mathbb{R}$, set $P^{1}(A)=$ $P\left(A \mid u_{k 1}, 1 \leq k \leq n\right)$, the conditional probability of $A$ with respect to $u_{k 1}, 1 \leq k \leq n$. For any $s>0$, from the fact that $\left\{u_{k 1}\right\},\left\{u_{k 2}\right\}$ and $\left\{u_{k 4}\right\}$ are independent, we see that

$$
\begin{aligned}
P\left(Z_{12}>a_{n}, Z_{14}>s a_{n}\right) & =E\left(P^{1}\left(Z_{12}>a_{n}\right) \cdot P^{1}\left(Z_{14}>s a_{n}\right)\right) \\
& \leq\left\{E P^{1}\left(Z_{12}>a_{n}\right)^{2}\right\}^{1 / 2} \cdot\left\{E P^{1}\left(Z_{14}>s a_{n}\right)^{2}\right\}^{1 / 2}
\end{aligned}
$$

by the Cauchy-Schwartz inequality. Taking $t_{n}:=a_{n} / \sqrt{n \log p} \rightarrow t=2$ and $t_{n}:=s a_{n} / \sqrt{n \log p} \rightarrow t=2 s$ in Lemma 6.7 , respectively, we get

$$
E P^{1}\left(Z_{12}>a_{n}\right)^{2}=O\left(p^{-4+\epsilon}\right) \text { and } E P^{1}\left(Z_{14}>s a_{n}\right)^{2}=O\left(p^{-4 s^{2}+\epsilon}\right)
$$

as $n \rightarrow \infty$ for any $\epsilon>0$. This implies that, for any $s>0$ and $\epsilon>0$,

$$
P\left(Z_{12}>a_{n}, Z_{14}>s a_{n}\right) \leq O\left(p^{-2-2 s^{2}+\epsilon}\right)
$$

as $n \rightarrow \infty$. In particular,

$$
P\left(Z_{12}>a_{n}, Z_{14}>\frac{a_{n}}{\sqrt{3}}\right) \leq O\left(p^{-\frac{8}{3}+\epsilon}\right)
$$

imsart-aos ver. 2010/08/03 file: Coherence_Supplement_01_15_11.tex date: January 15, 2011 
as $n \rightarrow \infty$ for any $\epsilon>0$.

Now we bound the last term in (122). Note that $\left|u_{11} u_{12}\right| \leq\left(u_{11}^{2}+u_{12}^{2}\right) / 2$, it follows that $E e^{\left|u_{11} u_{12}\right| / 2}<\infty$ by independence and $E \exp \left(N(0,1)^{2} / 4\right)<\infty$. Since $\left\{u_{k 1}, u_{k 2} ; 1 \leq k \leq n\right\}$ are i.i.d. with mean zero and variance one, and $y_{n}:=a_{n} / \sqrt{n \log p} \rightarrow 2$ as $n \rightarrow \infty$, taking $\alpha=1$ in Lemma 6.8 , we get

$$
\begin{aligned}
P\left(Z_{12}>a_{n}\right) & =P\left(\frac{1}{\sqrt{n \log p}}\left|\sum_{k=1}^{n} u_{k 1} u_{k 2}\right|>\frac{a_{n}}{\sqrt{n \log p}}\right) \\
& \sim 2 \cdot \frac{p^{-y_{n}^{2} / 2}(\log p)^{-1 / 2}}{2 \sqrt{2 \pi}} \sim \frac{e^{-y / 2}}{\sqrt{2 \pi}} \cdot \frac{1}{p^{2}}
\end{aligned}
$$

as $n \rightarrow \infty$. Similarly, for any $t>0$,

$$
P\left(Z_{12}>t a_{n}\right)=O\left(p^{-2 t^{2}+\epsilon}\right)
$$

as $n \rightarrow \infty$ (this can also be derived from (i) of Lemma 6.4). In particular,

$$
P\left(Z_{54}>\frac{a_{n}}{\sqrt{3}}\right)=P\left(Z_{12}>\frac{a_{n}}{\sqrt{3}}\right)=O\left(p^{-\frac{2}{3}+\epsilon}\right)
$$

as $n \rightarrow \infty$ for any $\epsilon>0$. Combining (125) and (127), we know that the last term in (122) is bounded by $O\left(p^{-\frac{8}{3}+\epsilon}\right)$ as $n \rightarrow \infty$ for any $\epsilon>0$. This together with (122) and (124) concludes the lemma.

Proof of Lemma 6.11. Fix $\delta \in(0,1)$. Take independent standard normals $\left\{u_{k 5}, u_{k 6} ; 1 \leq k \leq n\right\}$ that are also independent of $\left\{u_{k i} ; 1 \leq i \leq 4 ; 1 \leq k \leq\right.$ $n\}$. Then, since $\left\{u_{k 1}, u_{k 2}, u_{k 5}, u_{k 6} ; 1 \leq k \leq n\right\}$ are i.i.d. standard normals, by checking covariance matrix $\Sigma_{4}$, we know

$$
\left(u_{11}, u_{12}, u_{13}, u_{14}\right) \stackrel{d}{=}\left(u_{11}, u_{12}, r_{1} u_{11}+r_{1}^{\prime} u_{15}, r_{2} u_{12}+r_{2}^{\prime} u_{16}\right)
$$

where $r_{1}^{\prime}=\sqrt{1-r_{1}^{2}}$ and $r_{2}^{\prime}=\sqrt{1-r_{2}^{2}}$. Define $Z_{i j}=\left|\sum_{k=1}^{n} u_{k i} u_{k j}\right|$ for $1 \leq i, j \leq 6$. Then

$$
\begin{aligned}
& \left|\sum_{k=1}^{n}\left(r_{1} u_{k 1}+r_{1}^{\prime} u_{k 5}\right)\left(r_{2} u_{k 2}+r_{2}^{\prime} u_{k 6}\right)\right| \\
\leq & \left|r_{1} r_{2}\right| Z_{12}+\left|r_{1} r_{2}^{\prime}\right| Z_{16}+\left|r_{1}^{\prime} r_{2}\right| Z_{25}+\left|r_{1}^{\prime} r_{2}^{\prime}\right| Z_{56} \\
\leq & (1-\delta)^{2} Z_{12}+3 \max \left\{Z_{16}, Z_{25}, Z_{56}\right\}
\end{aligned}
$$

for all $\left|r_{1}\right|,\left|r_{2}\right| \leq 1-\delta$. Let $\alpha=\left(1+(1-\delta)^{2}\right) / 2, \beta=\alpha /(1-\delta)^{2}$ and $\gamma=(1-\alpha) / 3$. Then

$$
\beta>1 \text { and } \gamma>0 \text {. }
$$

imsart-aos ver. 2010/08/03 file: Coherence_Supplement_01_15_11.tex date: January 15, 2011 
Easily, if $Z_{12} \leq \beta a_{n}, \max \left\{Z_{16}, Z_{25}, Z_{56}\right\} \leq \gamma a_{n}$, then from (129) we know that the left hand side of (129) is controlled by $a_{n}$. Consequently, by (128) and the i.i.d. property,

$$
\begin{aligned}
& P\left(Z_{12}>a_{n}, Z_{34}>a_{n}\right) \\
= & P\left(Z_{12}>a_{n},\left|\sum_{k=1}^{n}\left(r_{1} u_{k 1}+r_{1}^{\prime} u_{k 5}\right)\left(r_{2} u_{k 2}+r_{2}^{\prime} u_{k 6}\right)\right|>a_{n}\right) \\
\leq & P\left(Z_{12}>a_{n}, Z_{12}>\beta a_{n}\right)+\sum_{i \in\{1,2,5\}} P\left(Z_{12}>a_{n}, Z_{i 6}>\gamma a_{n}\right) \\
= & P\left(Z_{12}>\beta a_{n}\right)+2 P\left(Z_{12}>a_{n}, Z_{16}>\gamma a_{n}\right) \\
+ & P\left(Z_{12}>a_{n}\right) \cdot P\left(Z_{56}>\gamma a_{n}\right)
\end{aligned}
$$

where " $2 P\left(Z_{12}>a_{n}, Z_{16}>\gamma a_{n}\right)$ " comes from that $\left(Z_{12}, Z_{16}\right) \stackrel{d}{=}\left(Z_{12}, Z_{26}\right)$. Keep in mind that $\left(Z_{12}, Z_{16}\right) \stackrel{d}{=}\left(Z_{12}, Z_{14}\right)$ and $Z_{56} \stackrel{d}{=} Z_{12}$. Recall (130), applying (123) and (126) to the three terms in the sum on the right hand side of (131), we conclude (72).

Proof of Lemma 6.12. Reviewing notation $\Omega_{3}=\Omega_{j}$ for $j=3$ defined below (65), the current case is that $d_{1} \leq d_{3} \leq d_{2} \leq d_{4}$ with $d=\left(d_{1}, d_{2}\right)$ and $d^{\prime}=\left(d_{3}, d_{4}\right)$. Of course, by definition, $d_{1}<d_{2}$ and $d_{3}<d_{4}$. To save notation, define the "neighborhood" of $d_{i}$ as follows:

$$
N_{i}=\left\{d \in\{1, \cdots, p\} ;\left|d-d_{i}\right|<\tau\right\}
$$

for $i=1,2,3,4$.

Given $d_{1}<d_{2}$, there are two possibilities for $d_{4}$ : (a) $d_{4}-d_{2}>\tau$ and (b) $0 \leq d_{4}-d_{2} \leq \tau$. There are four possibilities for $d_{3}$ : (A) $d_{3} \in N_{2} \backslash N_{1}$; (B) $d_{3} \in N_{1} \backslash N_{2}$; (C) $d_{3} \in N_{1} \cap N_{2}$; (D) $d_{3} \notin N_{1} \cup N_{2}$. There are eight combinations for the locations of $\left(d_{3}, d_{4}\right)$ in total. However, by (64) the combination (a) \& (D) is excluded. Our analysis next will exhaust all of the seven possibilities.

Case (a) \& (A). Let $\Omega_{a, A}$ be the subset of $\left(d, d^{\prime}\right) \in \Omega_{3}$ satisfying restrictions (a) and (A), and others such as $\Omega_{b, C}$ are similarly defined. Thus,

$$
\text { (133) } \sum_{\left(d, d^{\prime}\right) \in \Omega_{3}} P\left(Z_{d}>a_{n}, Z_{d^{\prime}}>a_{n}\right) \leq \sum_{\theta, \Theta} \sum_{\left(d, d^{\prime}\right) \in \Omega_{\theta, \Theta}} P\left(Z_{d}>a_{n}, Z_{d^{\prime}}>a_{n}\right)
$$

where $\theta$ runs over set $\{a, b\}$ and $\Theta$ runs over set $\{A, B, C, D\}$ but $(\theta, \Theta) \neq$ $(a, D)$. 
Easily, $\left|\Omega_{a, A}\right| \leq \tau p^{3}$ and the covariance matrix of $\left(w_{d_{2}}, w_{d_{1}}, w_{d_{3}}, w_{d_{4}}\right)$ (see $(67))$ is

$$
\left(\begin{array}{llll}
1 & 0 & \gamma & 0 \\
0 & 1 & 0 & 0 \\
\gamma & 0 & 1 & 0 \\
0 & 0 & 0 & 1
\end{array}\right), \quad|\gamma| \leq 1
$$

Take $\epsilon=1 / 2$ in Lemma 6.9 to have $P\left(Z_{d}>a_{n}, Z_{d^{\prime}}>a_{n}\right) \equiv \rho_{n}=o\left(p^{-7 / 2}\right)$ for all $\left(d, d^{\prime}\right) \in \Omega_{a, A}$. Thus

$$
\sum_{\left(d, d^{\prime}\right) \in R} P\left(Z_{d}>a_{n}, Z_{d^{\prime}}>a_{n}\right)=|R| \cdot \rho_{n} \rightarrow 0
$$

as $n \rightarrow \infty$ for $R=\Omega_{a, A}$.

Case (a) \& (B). Notice $\left|\Omega_{a, B}\right| \leq \tau p^{3}$ and $\left(w_{d_{1}}, w_{d_{2}}, w_{d_{3}}, w_{d_{4}}\right)$ has the same covariance matrix as that in Lemma 6.9. By the lemma we then have (134) for $R=\Omega_{a, B}$.

Case (a) \& (C). Notice $\left|\Omega_{a, C}\right| \leq \tau^{2} p^{2}$ and the covariance matrix of $\left(w_{d_{1}}, w_{d_{2}}, w_{d_{3}}, w_{d_{4}}\right)$ is the same as that in Lemma 6.10. By the lemma, we know (134) holds for $R=\Omega_{a, C}$.

Case (b) \& (A). In this case, $\left|\Omega_{b, A}\right| \leq \tau^{2} p^{2}$ and the covariance matrix of $\left(w_{d_{3}}, w_{d_{4}}, w_{d_{2}}, w_{d_{1}}\right)$ is the same as that in Lemma 6.10. By the lemma and using the fact that

$$
P\left(Z_{d}>a_{n}, Z_{d^{\prime}}>a_{n}\right)=P\left(Z_{\left(d_{3}, d_{4}\right)}>a_{n}, Z_{\left(d_{2}, d_{1}\right)}>a_{n}\right)
$$

we see (134) holds with $R=\Omega_{b, A}$.

Case (b) \& (B). In this case, $\left|\Omega_{b, B}\right| \leq \tau^{2} p^{2}$ and the covariance matrix of $\left(w_{d_{1}}, w_{d_{2}}, w_{d_{3}}, w_{d_{4}}\right)$ is the same as that in Lemma 6.11. By the lemma, we know (134) holds for $R=\Omega_{b, B}$.

Case (b) \& (C). We assign positions for $d_{1}, d_{3}, d_{2}, d_{4}$ step by step: there are at most $p$ positions for $d_{1}$ and at most $k$ positions for each of $d_{3}, d_{2}$ and $d_{4}$. Thus, $\left|\Omega_{b, C}\right| \leq \tau^{3} p$. By (125),

$$
P\left(Z_{d}>a_{n}, Z_{d^{\prime}}>a_{n}\right) \leq P\left(Z_{d}>a_{n}\right)=P\left(\left|\sum_{i=1}^{n} \xi_{i} \eta_{i}\right|>a_{n}\right)=O\left(\frac{1}{p^{2}}\right)
$$

as $n \rightarrow \infty$, where $\left\{\xi_{i}, \eta_{i} ; i \geq 1\right\}$ are i.i.d. standard normals. Therefore, (134) holds with $R=\Omega_{b, C}$. 
Case (b) \& (D). In this case, $\left|\Omega_{b, C}\right| \leq \tau p^{3}$ and the covariance matrix of $\left(w_{d_{4}}, w_{d_{3}}, w_{d_{2}}, w_{d_{1}}\right)$ is the same as that in Lemma 6.9. By the lemma and noting the fact that

$$
P\left(Z_{d}>a_{n}, Z_{d^{\prime}}>a_{n}\right)=P\left(Z_{\left(d_{4}, d_{3}\right)}>a_{n}, Z_{\left(d_{2}, d_{1}\right)}>a_{n}\right)
$$

we see (134) holds with $R=\Omega_{b, D}$.

We obtain (72) by combining (134) for all the cases considered above with (133).

Department of Statistics

The Wharton SCHOOL

University of Pennsylvania

Philadelphia, PA 19104.

E-MAIL: tcai@wharton.upenn.edu

URL: http://www-stat.wharton.upenn.edu/ tcai
SCHOOL OF Statistics

UNIVERSiTy OF MiNNESOTA

224 Church Street

MinNEAPOLIS, MN55455

E-MAIL: tjiang@stat.umn.edu

URL: http://www.stat.umn.edu/ tjiang 